\title{
Characterization of quantum angular-momentum fluctuations via principal components
}

\author{
Ángel Rivas and Alfredo Luis* \\ Departamento de Óptica, Facultad de Ciencias Físicas, Universidad Complutense, 28040 Madrid, Spain
}

(Received 7 October 2007; published 12 February 2008)

\begin{abstract}
We elaborate an approach to quantum fluctuations of angular momentum based on the diagonalization of the covariance matrix in two versions: real symmetric and complex Hermitian. At difference with previous approaches this is $\mathrm{SU}(2)$ invariant and avoids any difficulty caused by nontrivial commutators. Meaningful uncertainty relations are derived which are nontrivial even for vanishing mean angular momentum. We apply this approach to some relevant states.
\end{abstract}

DOI: 10.1103/PhysRevA.77.022105

PACS number(s): 03.65.Ca, 42.50.St, 42.25.Ja

\section{INTRODUCTION}

Quantum fluctuations and uncertainty relations play a key role in the fundamentals of quantum physics and its applications. In this work we focus on angular momentum variables. Beside mechanics, angular momentum operators are ubiquitous in areas such as quantum optics, matter-light interactions, and Bose-Einstein condensates. Basic observables such as light intensity, number of particles, and atomic populations are formally equivalent to angular-momentum components [1-5]. This is also the case of the Stokes parameters representing light polarization and the internal state of twolevel atoms identified as spin-1/2 systems [5-7]. Angularmomentum operators are also the generators of basic operations such as phase shifting, beam splitting, free evolution, as well as atomic interactions with classical fields $[7,8]$.

Moreover, angular-momentum fluctuations are crucial in diverse areas. This is, for example, the case of quantum metrology, implemented by very different physical systems such as two beam interference with light or Bose-Einstein condensates, or atomic population spectroscopy. This is because angular-momentum uncertainty relations determine the ultimate limit to the resolution of interferometric and spectroscopic measurements [1-3]. A dramatic example has been put forward in Ref. [3] concerning atomic clocks based on atomic population spectroscopy, whose signal to noise ratio is proportional to the square root of the duration of the measurement. In such a case, an atomic clock using $10^{10}$ atoms prepared in a state with reduced angular-momentum fluctuations would yield the same signal to noise ratio in a measurement lasting $1 \mathrm{~s}$ as an atomic clock with the same number of atoms in a state without reduced angular-momentum fluctuations in a measurement lasting 300 yr. From a different perspective it has been shown that angular-momentum fluctuations are useful in the analysis of many-body entanglement [4] and continuous-variable polarization entanglement [5].

In comparison to other fundamental variables, such as position and momentum, the standard uncertainty relations for angular momentum run into two serious difficulties: basic commutators are operators instead of numbers, and there is lack of $\mathrm{SU}(2)$ invariance. Nontrivial commutation relations lead to uncertainty products bounded by state-dependent

\footnotetext{
*alluis@ fis.ucm.es; URL: http://www.ucm.es/info/gioq
}

quantities. Among other consequences, these bounds become trivial for states with vanishing mean angular momentum. On the other hand, lack of SU(2) invariance is a basic difficulty since two states connected by a $\mathrm{SU}(2)$ transformation should be equivalent concerning quantum fluctuations, in the same sense that phase-space displacements are irrelevant for position-momentum uncertainty relations.

In this work we elaborate an approach to quantum fluctuations for angular-momentum variables, which is $\mathrm{SU}(2)$ invariant and avoids the difficulties caused by nontrivial commutators. The analysis is based on the diagonalization of the covariance matrix, which we study in two versions: real symmetric (Sec. III) and complex Hermitian (Sec. IV). We examine the main properties of their eigenvalues (principal variances) and eigenvectors (principal components). In Sec. $\mathrm{V}$ we derive uncertainty relations involving principal variances that are meaningful even in the case of vanishing mean values. Finally, in Sec. VI we illustrate this approach applying it to some relevant examples. Section II is devoted to recalling basic concepts and definitions.

\section{ANGULAR-MOMENTUM OPERATORS AND SU(2) INVARIANCE}

Let us consider arbitrary dimensionless angularmomentum operators $\mathbf{j}=\left(j_{1}, j_{2}, j_{3}\right)$ satisfying the commutation relations

$$
\left[j_{k}, j_{\ell}\right]=i \sum_{n=1}^{3} \epsilon_{k, \ell, n} j_{n}, \quad\left[j_{0}, \mathbf{j}\right]=\mathbf{0},
$$

where $\epsilon_{k, \ell, n}$ is the fully antisymmetric tensor with $\epsilon_{1,2,3}=1$, and $j_{0}$ is defined by the relation

$$
\mathbf{j}^{2}=j_{0}\left(j_{0}+1\right) \text {. }
$$

For the sake of completeness we take into account that $j_{0}$ may be an operator. This is the case of two-mode bosonic realizations where $j_{0}$ is proportional to the number of particles.

More specifically, denoting by $a_{1,2}$ the annihilation operators of two independent bosonic modes with $\left[a_{j}, a_{j}^{\dagger}\right]=1$, $\left[a_{1}, a_{2}\right]=\left[a_{1}, a_{2}^{\dagger}\right]=0$, we get that the following operators

$$
j_{0}=\frac{1}{2}\left(a_{1}^{\dagger} a_{1}+a_{2}^{\dagger} a_{2}\right), \quad j_{1}=\frac{1}{2}\left(a_{2}^{\dagger} a_{1}+a_{1}^{\dagger} a_{2}\right),
$$




$$
j_{2}=\frac{i}{2}\left(a_{2}^{\dagger} a_{1}-a_{1}^{\dagger} a_{2}\right), \quad j_{3}=\frac{1}{2}\left(a_{1}^{\dagger} a_{1}-a_{2}^{\dagger} a_{2}\right),
$$

satisfy Eqs. (2.1) and (2.2) [9]. Concerning physical realizations, $a_{1,2}$ can represent the complex amplitude operators of two electromagnetic field modes. For material systems they can represent the annihilation operators for two species of atoms in two different internal states, for example. In any case, the operator $j_{0}$ is proportional to the total number of photons or atoms and $j_{3}$ is proportional to the number difference. Therefore, these operators represent basic detection mechanisms such as the measurement of light intensity or the number of atoms. In this regard we have the following correspondence:

$$
|j, m\rangle=\left|n_{1}=j+m\right\rangle\left|n_{2}=j-m\right\rangle,
$$

between the standard angular-momentum basis $|j, m\rangle$ of simultaneous eigenvectors of $j_{3}$ and $j_{0}$, i.e., $j_{3}|j, m\rangle=m|j, m\rangle$, $j_{0}|j, m\rangle=j|j, m\rangle$, and the product of number states in the two modes $\left|n_{1}\right\rangle\left|n_{2}\right\rangle$ with $a_{j}^{\dagger} a_{j}\left|n_{j}\right\rangle=n_{j}\left|n_{j}\right\rangle$.

Angular-momentum operators also serve to describe the internal state of two-level atoms via the definitions

$$
\begin{aligned}
& j_{0}=\frac{1}{2}(|e\rangle\langle e|+| g\rangle\langle g|), \quad j_{1}=\frac{1}{2}(|g\rangle\langle e|+| e\rangle\langle g|), \\
& j_{2}=\frac{i}{2}(|g\rangle\langle e|-| e\rangle\langle g|), \quad j_{3}=\frac{1}{2}(|e\rangle\langle e|-| g\rangle\langle g|),
\end{aligned}
$$

where $|e, g\rangle$ are the excited and ground states. This is formally a spin- $1 / 2$ system where $j_{0,3}$ represents atomic populations and $j_{1,2}$ represents the atomic dipole [7]. Collections of two-level atoms are described by the composition of the individual angular momenta.

Throughout, by SU(2) invariance we mean that the density operators $\rho$ and $U \rho U^{\dagger}$ are fully equivalent concerning quantum fluctuations, where $U$ is any $\mathrm{SU}(2)$ unitary operator exponential of the angular-momentum operators

$$
U=\exp (i \theta \mathbf{u} \cdot \mathbf{j}),
$$

with $\theta$ a real parameter, and $\mathbf{u}$ a unit three-dimensional real vector. It can be seen that the action of $U$ on $\mathbf{j}$ is a rotation $R$ of angle $\theta$ and axis $\mathbf{u}[10]$,

$$
U^{\dagger} \mathbf{j} U=R \mathbf{j},
$$

where $R^{t} R=R R^{t}=I, I$ is the $3 \times 3$ identity, and the superscript $t$ denotes matrix transposition. So, the $\mathrm{SU}(2)$ invariance is just the mathematical statement corresponding to the fact that the conclusions which one could draw from an angularmomentum measurement must be independent of which set of three orthogonal angular-momentum components one chooses.

One way to guarantee the cited invariance is obtained by using specific components of the angular momentum defined in terms of the mean value $\langle\mathbf{j}\rangle$, the longitudinal $j_{\|}$, and transversal $j_{\perp, k}$ components with $k=1,2$. These components are the projections of $\mathbf{j}$ on a set of Cartesian axes adapted to $\langle\mathbf{j}\rangle$ so that the longitudinal axis points in the direction of $\langle\mathbf{j}\rangle$ $[11,12]$. Therefore, by construction

$$
|\langle\mathbf{j}\rangle|=\left\langle j_{\|}\right\rangle, \quad\left\langle j_{\perp, k}\right\rangle=0 .
$$

Among other properties $j_{\|}, j_{\perp}$ serve to properly define $\mathrm{SU}(2)$ squeezing as reduced fluctuations of a transversal component $[1,11]$. However, this approach breaks down in states with vanishing mean angular momentum.

Finally, we recall a basic relation showing that angularmomentum fluctuations limit the resolution of interferometric and spectroscopic measurements [1-3]. This is because angular-momentum components $j_{n}$ describe atomic and light free evolution, as well as the action of linear optical devices such as beam splitters and interferometers, via unitary transformations of the form $U_{\phi}=\exp \left(i \phi j_{n}\right)$ acting in a given initial state $\rho$. The objective of interferometric and spectroscopic measurements is the accurate determination of the value of the phase shift $\phi$. This is carried out by the measurement of a given observable $A$ in the transformed state $U_{\phi} \rho U_{\phi}^{\dagger}$. In a simple data analysis, the uncertainty $\Delta \phi$ in the inferred value of $\phi$ is related to the fluctuations of the measured observable in the form [1]

$$
\Delta \phi=\left|\frac{\partial\langle A\rangle}{\partial \phi}\right|^{-1} \Delta A=\frac{\Delta A}{\left|\left\langle\left[A, j_{n}\right]\right\rangle\right|} \geqslant \frac{1}{2 \Delta j_{n}},
$$

where the uncertainty relation $\Delta A \Delta j_{n} \geqslant\left|\left\langle\left[A, j_{n}\right]\right\rangle\right| / 2$ has been used. Therefore, the accuracy of the detection is limited by the fluctuations of the angular-momentum component generating the transformation. In the optimum case $\Delta j_{n} \propto\left\langle j_{0}\right\rangle$ so that $\Delta \phi$ is inversely proportional to the mean number of particles. This ultimate limit is known as Heisenberg limit [1]. It is worth stressing that this conclusion applies exclusively to pure states, as required by the equality in the uncertainty product between $A$ and $j_{n}$. These results are supported by more involved analyses [13].

\section{PRINCIPAL COMPONENTS FOR REAL SYMMETRIC COVARIANCE MATRIX}

Complete second-order statistics of the operators $\mathbf{j}$ in a given state $\rho$ is contained in the $3 \times 3$ covariance matrix associated with $\rho$. Due to the lack of commutativity we can propose two different covariance matrices: real symmetric and complex Hermitian.

In this section we focus on the real symmetric covariance $3 \times 3$ matrix $M$ with matrix elements

$$
M_{k, \ell}=\frac{1}{2}\left(\left\langle j_{k} j_{\ell}\right\rangle+\left\langle j_{\ell} j_{k}\right\rangle\right)-\left\langle j_{k}\right\rangle\left\langle j_{\ell}\right\rangle,
$$

where mean values are taken with respect to $\rho$ and we have $M_{k, \ell}^{*}=M_{k, \ell}=M_{\ell, k}$. Next we analyze the main properties of $M$.

(i) The covariance matrix $M$ allows us to compute the variances $\left(\Delta j_{u}\right)^{2}$ of arbitrary angular-momentum components $j_{u}=\mathbf{u} \cdot \mathbf{j}$, where $\mathbf{u}$ is any unit real vector, in the form

$$
\left(\Delta j_{u}\right)^{2}=\mathbf{u}^{t} M \mathbf{u} .
$$

Similarly, $M$ allows us to compute the symmetric correlation of two arbitrary components $j_{u}=\mathbf{u} \cdot \mathbf{j}, j_{v}=\mathbf{v} \cdot \mathbf{j}$, where $\mathbf{u}, \mathbf{v}$ are unit real vectors, in the form 


$$
\frac{1}{2}\left(\left\langle j_{u} j_{v}\right\rangle+\left\langle j_{v} j_{u}\right\rangle\right)-\left\langle j_{u}\right\rangle\left\langle j_{v}\right\rangle=\mathbf{v}^{t} M \mathbf{u}=\mathbf{u}^{t} M \mathbf{v} .
$$

(ii) Since $M$ is real symmetric the transformation that renders $M$ diagonal is a rotation matrix $R_{d}$,

$$
M=R_{d}^{t}\left(\begin{array}{ccc}
\left(\Delta J_{1}\right)^{2} & 0 & 0 \\
0 & \left(\Delta J_{2}\right)^{2} & 0 \\
0 & 0 & \left(\Delta J_{3}\right)^{2}
\end{array}\right) R_{d} .
$$

The eigenvalues $\left(\Delta J_{k}\right)^{2}, k=1,2,3$, are the variances of the operators $J_{k}=\mathbf{u}_{k} \cdot \mathbf{j}$, where $\mathbf{u}_{k}$ are the three real orthonormal eigenvectors of $M$,

$$
M \mathbf{u}_{k}=\left(\Delta J_{k}\right)^{2} \mathbf{u}_{k} .
$$

This implies that $M$ is a positive semidefinite matrix. Following standard nomenclature in statistics we refer to $\mathbf{J}$ and $\Delta \mathbf{J}=\left(\Delta J_{1}, \Delta J_{2}, \Delta J_{3}\right)$ as principal components and variances, respectively. We stress that $\mathbf{J}$ and $\Delta \mathbf{J}$ depend on the system state $\rho$. lated,

(iii) For every $\rho$ the principal components are uncorre-

$$
\frac{1}{2}\left(\left\langle J_{k} J_{\ell}\right\rangle+\left\langle J_{\ell} J_{k}\right\rangle\right)-\left\langle J_{k}\right\rangle\left\langle J_{\ell}\right\rangle=0, \quad k \neq \ell .
$$

(iv) The principal components $\mathbf{J}$ are related to $\mathbf{j}$ by the rotation $R_{d}$ that diagonalizes $M$,

$$
\mathbf{J}=R_{d} \mathbf{j} .
$$

Thus, the three operators $\mathbf{J}$ are legitimate mutually orthogonal Hermitian angular-momentum components satisfying the standard commutation relations

$$
\left[J_{k}, J_{\ell}\right]=i \sum_{n=1}^{3} \epsilon_{k, \ell, n} J_{n}
$$

and

$$
\mathbf{J}^{2}=\mathbf{j}^{2}, \quad\langle\mathbf{J}\rangle^{2}=\langle\mathbf{j}\rangle^{2} .
$$

(v) At most one principal variance can vanish for $j_{0} \neq 0$ since for $j_{0} \neq 0$ no state can be an eigenvector of more than one angular-momentum component.

(vi) The principal variances provide an SU(2) invariant characterization of fluctuations. The invariance holds because under any SU(2) transformation (2.6) and (2.7) we get that $M$ transforms as $M \rightarrow R M R^{t}$. Therefore, the covariance matrix $R M R^{t}$ associated to the state $U^{\dagger} \rho U$ has the same principal variances as the covariance matrix $M$ associated to the state $\rho$.

(vii) The principal variances are the extremes of the variances of arbitrary angular-momentum components $j_{u}=\mathbf{u} \cdot \mathbf{j}$ for fixed $\rho$ when the real unit vector $\mathbf{u}$ is varied. More specifically, from Eq. (3.2), taking into account that $M$ is symmetric, and using a Lagrange multiplier $\lambda$ for the constraint $\mathbf{u}^{2}=1$, we have that the extremes of $\Delta j_{u}$ when $\mathbf{u}$ is varied are given by the eigenvalue equation

$$
M \mathbf{u}=\lambda \mathbf{u},
$$

so that from Eq. (3.5) the extremes coincide with the principal components.

(viii) Next we examine the relation between the principal components and the longitudinal $j_{\|}$and transversal $j_{\perp, k}$ components, with $k=1,2$. We can demonstrate that $j_{\|}$is a principal component when the state $\rho$ is invariant $U \rho U^{\dagger}=\rho$ under the unitary transformation $U=\exp \left(i \pi j_{\|}\right)$. This is because for this transformation

$$
U^{\dagger} j_{\perp, k} U=-j_{\perp, k}, \quad U^{\dagger} j_{\|} U=j_{\|},
$$

and

$$
U^{\dagger} j_{\perp, k} j_{\|} U=-j_{\perp, k} j_{\|}
$$

so that

$$
U \rho U^{\dagger}=\rho \rightarrow\left\langle j_{\perp, k} j_{\|}\right\rangle=0,
$$

and similarly for the opposite ordering $\left\langle j_{\|} j_{\perp, k}\right\rangle=0$. Then $j_{\|}$is a principal component and the other two principal components are transversal.

Invariance under $U=\exp \left(i \pi j_{\|}\right)$is a very frequent symmetry. For bosonic two-mode realizations this is equivalent to symmetry under mode exchange $a_{1} \leftrightarrow a_{2}$ for the modes for which $j_{\|}=\left(a_{1}^{\dagger} a_{2}+a_{2}^{\dagger} a_{1}\right) / 2$, where $a_{1,2}$ are the corresponding annihilation operators. This is because for these modes $U^{\dagger} a_{1} U=i a_{2}$ and $U^{\dagger} a_{2} U=i a_{1}$ and we have mode exchange except for a global $\pi / 2$ phase change.

(ix) According to Eq. (2.9) the maximum principal variance of $\rho$ provides an assessment of the resolution achievable with $\rho$ in the detection of small phase shifts generated by angular-momentum components. This includes all linear interferometric and spectroscopic measurements. Therefore, the maximum principal variance provides an estimation of the usefulness of the corresponding state in quantum metrology.

(x) Next we derive upper and lower bounds for the principal variances of states with $\langle\mathbf{j}\rangle=\mathbf{0}$. In such a case from Eqs. (2.2) and (3.9) we have

$$
\operatorname{tr} M=\left(\Delta J_{1}\right)^{2}+\left(\Delta J_{2}\right)^{2}+\left(\Delta J_{3}\right)^{2}=\left\langle j_{0}\left(j_{0}+1\right)\right\rangle .
$$

If we arrange the principal variances in decreasing order $\Delta J_{1} \geqslant \Delta J_{2} \geqslant \Delta J_{3}$, and using Eq. (3.14) we get the following bounds for the principal variances:

$$
\begin{gathered}
\left\langle j_{0}^{2}\right\rangle \geqslant\left(\Delta J_{1}\right)^{2} \geqslant \frac{1}{3}\left\langle j_{0}\left(j_{0}+1\right)\right\rangle, \\
\frac{1}{2}\left\langle j_{0}\left(j_{0}+1\right)\right\rangle \geqslant\left(\Delta J_{2}\right)^{2} \geqslant \frac{1}{2}\left\langle j_{0}\right\rangle, \\
\frac{1}{3}\left\langle j_{0}\left(j_{0}+1\right)\right\rangle \geqslant\left(\Delta J_{3}\right)^{2} \geqslant 0 .
\end{gathered}
$$

The upper bound for $\Delta J_{1}$ holds because for an arbitrary component $\left\langle j_{k}^{2}\right\rangle \leqslant\left\langle j_{0}^{2}\right\rangle$, while the lower bound is reached when all the principal variances are equal. The upper bound for $\Delta J_{2}$ is reached when $\Delta J_{1}=\Delta J_{2}$ and $\Delta J_{3}=0$, while the lower bound 
is reached when $\left(\Delta J_{1}\right)^{2}=\left\langle j_{0}^{2}\right\rangle$ and $\Delta J_{2}=\Delta J_{3}$. Finally, the upper bound for $\Delta J_{3}$ is reached when all the principal variances are equal, while the lower bound occurs for $\Delta J_{3}=0$.

(xi) The lower bound for $\Delta J_{1}$ in Eq. (3.15) is, roughly speaking, of the order of $\left\langle j_{0}\right\rangle$ so that from Eq. (2.9) all pure states with $\langle\mathbf{j}\rangle=\mathbf{0}$ can reach maximum interferometric precision (Heisenberg limit). This explains why most optimum states for metrological applications satisfy $\langle\mathbf{j}\rangle=\mathbf{0}$ (see Sec. $\mathrm{VI})$.

\section{PRINCIPAL COMPONENTS FOR COMPLEX HERMITIAN COVARIANCE MATRIX}

In this section we elaborate the statistical description of angular-momentum fluctuations via the complex Hermitian $3 \times 3$ covariance matrix $\tilde{M}$ with matrix elements

$$
\tilde{M}_{k, \ell}=\left\langle j_{k} j_{\ell}\right\rangle-\left\langle j_{k}\right\rangle\left\langle j_{\ell}\right\rangle,
$$

with $\tilde{M}_{k, \ell}^{*}=\tilde{M}_{\ell, k}$. The two matrices $M$ and $\tilde{M}$ contain essentially the same information since they only differ by a factor $\langle\mathbf{j}\rangle$,

$$
M_{k, \ell}=\tilde{M}_{k, \ell}-\frac{i}{2} \sum_{n=1}^{3} \epsilon_{k, \ell, n}\left\langle j_{n}\right\rangle .
$$

In particular, they coincide exactly for all states with $\langle\mathbf{j}\rangle=\mathbf{0}$.

Nevertheless, $M$ and $\tilde{M}$ are different matrices so we can exploit this difference by examining the most relevant features that they do not share in common.

(i) The covariance matrix $\tilde{M}$ provides the properly defined variances of complex linear combinations of angularmomentum components $j_{u}=\mathbf{u} \cdot \mathbf{j}$ for arbitrary unit complex vectors $\mathbf{u}$ with $\mathbf{u}^{\dagger} \mathbf{u}=1$.

More specifically, for $\mathbf{u}^{*} \neq \mathbf{u}$ we have that $j_{u}$ is not Hermitian, $j_{u}^{\dagger} \neq j_{u}$, so that the variance must be redefined, for example, in the form [14]

$$
\left(\Delta j_{u}\right)^{2}=\left\langle j_{u}^{\dagger} j_{u}\right\rangle-\left|\left\langle j_{u}\right\rangle\right|^{2} .
$$

Then $\tilde{M}$ provides $\Delta j_{u}$ as

$$
\left(\Delta j_{u}\right)^{2}=\mathbf{u}^{\dagger} \tilde{M} \mathbf{u} .
$$

Note that for real $\mathbf{u}$ we have

$$
\mathbf{u}^{\dagger} \tilde{M} \mathbf{u}=\mathbf{u}^{t} M \mathbf{u} .
$$

Similarly, we can compute the correlation of two complex projections $j_{u}=\mathbf{u} \cdot \mathbf{j}, j_{v}=\mathbf{v} \cdot \mathbf{j}$, where $\mathbf{u}, \mathbf{v}$ are arbitrary unit complex vectors, in the form

$$
\left\langle j_{v}^{\dagger} j_{u}\right\rangle-\left\langle j_{v}^{\dagger}\right\rangle\left\langle j_{u}\right\rangle=\mathbf{v}^{\dagger} \tilde{M} \mathbf{u} .
$$

(ii) Since $\tilde{M}$ is Hermitian it becomes diagonal by means of a $3 \times 3$ unitary matrix $\mathcal{U}_{d}$,

$$
\tilde{M}=\mathcal{U}_{d}^{\dagger}\left(\begin{array}{ccc}
\left(\Delta \widetilde{J}_{1}\right)^{2} & 0 & 0 \\
0 & \left(\Delta \widetilde{J}_{2}\right)^{2} & 0 \\
0 & 0 & \left(\Delta \widetilde{J}_{3}\right)^{2}
\end{array}\right) \mathcal{U}_{d},
$$

where the elements on the diagonal are the variances (4.3) of the operators $\widetilde{J}_{k}=\mathbf{u}_{k} \cdot \mathbf{j}$, where $\mathbf{u}_{k}$ are the three complex orthonormal eigenvectors of $\tilde{M}$,

$$
\tilde{M} \mathbf{u}_{k}=\left(\Delta \widetilde{J}_{k}\right)^{2} \mathbf{u}_{k} .
$$

This implies that $\tilde{M}$ is positive semidefinite. We again refer to $\widetilde{\mathbf{J}}$ and $\Delta \widetilde{\mathbf{J}}=\left(\Delta \widetilde{J}_{1}, \Delta \widetilde{J}_{2}, \Delta \widetilde{J}_{3}\right)$ as principal components and variances, respectively. Since a global phase is irrelevant we regard always $\mathcal{U}_{d}$ as an $\mathrm{SU}(3)$ matrix.

(iii) For every state $\rho$ the principal components are uncorrelated,

$$
\left\langle\widetilde{J}_{k}^{\dagger} \tilde{J}_{\ell}\right\rangle-\left\langle\widetilde{J}_{k}^{\dagger}\right\rangle\left\langle\widetilde{J}_{\ell}\right\rangle=0, \quad k \neq \ell .
$$

(iv) Standard commutation relations (2.1) are not preserved under transformations by unitary $3 \times 3$ matrices,

$$
\widetilde{\mathbf{J}}=\mathcal{U} \mathbf{j} .
$$

However, a slight modification of Eq. (2.1) is actually preserved under the action (4.10) of SU(3) matrices. These are

$$
\left[\widetilde{J}_{k}, \widetilde{J}_{\ell}\right]=i \sum_{n=1}^{3} \epsilon_{k, \ell, n} \widetilde{J}_{n}^{\dagger}, \quad\left[j_{0}, \widetilde{\mathbf{J}}\right]=\mathbf{0},
$$

which are equivalent to Eq. (2.1) for Hermitian operators. We have also

$$
\tilde{\mathbf{J}}^{\dagger} \cdot \widetilde{\mathbf{J}}=\mathbf{j}^{2}, \quad\langle\widetilde{\mathbf{J}}\rangle^{*} \cdot\langle\widetilde{\mathbf{J}}\rangle=\langle\mathbf{j}\rangle^{2} .
$$

The preservation of Eq. (4.11) under the action of $\mathrm{SU}(3)$ matrices in Eq. (4.10) can be demonstrated by direct computation of the commutators after expressing the most general $\mathrm{SU}(3)$ matrix $\mathcal{U}$ as a suitable product of matrices belonging to the $\mathrm{SU}(2)$ subgroup of the form [15]

$$
\mathcal{U}=\left(\begin{array}{ccc}
\cos \theta e^{i \phi} & \sin \theta e^{i \varphi} & 0 \\
-\sin \theta e^{-i \varphi} & \cos \theta e^{-i \phi} & 0 \\
0 & 0 & 1
\end{array}\right),
$$

and permutations of lines and columns. Each matrix (4.13) transforms operators $\widetilde{\mathbf{J}}$ satisfying Eq. (4.11) into another set of operators $\widetilde{\mathbf{J}}^{\prime}=\mathcal{U} \tilde{\mathbf{J}}$ fulfilling the same commutation relations (4.11).

(v) From Eq. (4.2) we can derive the equality of traces $\operatorname{tr} M=\operatorname{tr} \tilde{M}$ so that

$$
(\Delta \widetilde{\mathbf{J}})^{2}=(\Delta \mathbf{J})^{2}=(\Delta \mathbf{j})^{2}=\left\langle j_{0}\left(j_{0}+1\right)\right\rangle-\langle\mathbf{J}\rangle^{2},
$$

with

$$
|\langle\widetilde{\mathbf{J}}\rangle|^{2}=\langle\mathbf{J}\rangle^{2}=\langle\mathbf{j}\rangle^{2} .
$$

Since $\langle\mathbf{J}\rangle^{2} \leqslant\left\langle j_{0}\right\rangle^{2}$ we have $(\Delta \widetilde{\mathbf{J}})^{2}>0$. This implies that there must be at least a nonvanishing principal variance. Other- 
wise, two principal variances of $\tilde{M}$ can vanish simultaneously for the same state, as shown in Sec. VI for the SU(2) coherent states.

(vi) Although the traces of $M$ and $\tilde{M}$ are equal the determinants are different. This can be easily proven by expressing $\tilde{M}$ in the principal-component basis that renders $M$ diagonal,

$$
\tilde{M}=\left(\begin{array}{ccc}
\left(\Delta J_{1}\right)^{2} & i\left\langle J_{3}\right\rangle / 2 & -i\left\langle J_{2}\right\rangle / 2 \\
-i\left\langle J_{3}\right\rangle / 2 & \left(\Delta J_{2}\right)^{2} & i\left\langle J_{1}\right\rangle / 2 \\
i\left\langle J_{2}\right\rangle / 2 & -i\left\langle J_{1}\right\rangle / 2 & \left(\Delta J_{3}\right)^{2}
\end{array}\right),
$$

so that

$$
\operatorname{det} \tilde{M}=\operatorname{det} M-\frac{1}{4} \sum_{k=1}^{3}\left(\Delta J_{k}\right)^{2}\left\langle J_{k}\right\rangle^{2} \text {, }
$$

and $\operatorname{det} M \geqslant \operatorname{det} \tilde{M}$.

(vii) The principal variances of $\tilde{M}$ are $\mathrm{SU}(2)$ invariant since under $\mathrm{SU}(2)$ transformations we have $\tilde{M} \rightarrow R \tilde{M} R^{t}$, so that the covariance matrix $R \tilde{M} R^{t}$ for the state $U^{\dagger} \rho U$ has the same eigenvalues as the covariance matrix $\tilde{M}$ for the state $\rho$.

(viii) The principal variances are the extremes of the variances $\left(\Delta j_{u}\right)^{2}$ of any complex combination of angularmomentum components $j_{u}=\mathbf{u} \cdot \mathbf{j}$, where $\mathbf{u}$ is a complex unit vector. More specifically, from Eq. (4.4), and introducing a Lagrange multiplier $\lambda$ to take into account the constraint $\mathbf{u}^{\dagger} \cdot \mathbf{u}=1$, we get that the extremes of $\Delta j_{u}$ are given by the eigenvalue equation

$$
\tilde{M} \mathbf{u}=\lambda \mathbf{u}
$$

so that from Eq. (4.8) the extremes of $\left(\Delta j_{u}\right)^{2}$ are the principal variances $\Delta \widetilde{\mathbf{J}}$.

(ix) The principal variances of $\tilde{M}$ are more extreme than the principal variances of $M$ since from Eq. (4.5) the variation process for the complex Hermitian case takes place over a larger set of operators $j_{u}$, with complex $\mathbf{u}$, that includes as a particular case the projections on real $\mathbf{u}$.

(x) Because of Eqs. (4.14) and (4.15) for $\langle\mathbf{j}\rangle=\mathbf{0}$ the upper and lower bounds for principal variances in Eq. (3.15) also hold replacing $\mathbf{J}$ by $\widetilde{\mathbf{J}}$.

(xi) It is questionable whether $\Delta j_{u}$ for $j_{u}^{\dagger} \neq j_{u}$ represents practical observable fluctuations. For example, for $j_{u}=j_{1}$ $+i j_{2}$ we have

$$
\left(\Delta j_{u}\right)^{2}=\left(\Delta j_{1}\right)^{2}+\left(\Delta j_{2}\right)^{2}-\left\langle j_{3}\right\rangle
$$

so we can have $\Delta j_{u}=0$ with $\Delta j_{1,2} \neq 0$, being this the case of the SU(2) coherent states (see Sec. VI). Nevertheless, from Eq. (4.14) we have that $\Delta \widetilde{\mathbf{J}}$ contains all angular-momentum fluctuations.

In this regard it is worth recalling that non-Hermitian operators can be related to experimental processes, as demonstrated by double homodyne detection where the statistics is given by projection on quadrature coherent states [16]. In our context, the eigenstates of $j_{u}=j_{1}+i j_{2}$ are $\mathrm{SU}(2)$ coherent states that define by projection the $\mathrm{SU}(2) Q$ function [10].
This is an observable probability distribution function, via double homodyne detection of two field modes for example [17].

\section{UNCERTAINTY RELATIONS}

Variances are the most popular building blocks of uncertainty relations. For angular momentum, the standard procedure leads to

$$
\Delta j_{1} \Delta j_{2} \geqslant \frac{1}{2}\left|\left\langle j_{3}\right\rangle\right|
$$

and cyclic permutations. This uncertainty relation faces two difficulties. On the one hand it is bounded by a statedependent quantity that vanish for states with $\langle\mathbf{j}\rangle=\mathbf{0}$. On the other hand, it lacks $S U(2)$ invariance so that it leads to different conclusions when applied to $\mathrm{SU}(2)$ equivalent states [18]. These difficulties can be avoided by using the principal variances leading to meaningful $\mathrm{SU}(2)$ invariant relations which are nontrivial even for states with $\langle\mathbf{j}\rangle=\mathbf{0}$.

A first $\mathrm{SU}(2)$ invariant uncertainty relation can be derived from the trace of $M$ (or equivalently $\tilde{M}$ ) [19]

$$
\operatorname{tr} M=(\Delta \mathbf{J})^{2}=\left\langle j_{0}\left(j_{0}+1\right)\right\rangle-\langle\mathbf{J}\rangle^{2} \geqslant\left\langle j_{0}\right\rangle,
$$

where we have used that for any component $\left\langle j_{k}\right\rangle^{2} \leqslant\left\langle j_{k}^{2}\right\rangle$ $\leqslant\left\langle j_{0}^{2}\right\rangle$.

The minimum uncertainty states with $(\Delta \mathbf{J})^{2}=\left\langle j_{0}\right\rangle$ are obtained for maximum $\langle\mathbf{J}\rangle^{2}=\left\langle j_{0}^{2}\right\rangle$. This is satisfied exclusively by $\mathrm{SU}(2)$ coherent states $[10,19]$

$$
|j, \theta, \mathbf{u}\rangle=U(\theta, \mathbf{u})|j, j\rangle,
$$

where $U$ is any $\mathrm{SU}(2)$ unitary operator (2.6) and $|j, m\rangle$ are the simultaneous eigenvectors of $j_{0}$ and $j_{3}$, with eigenvalues $j$ and $m$, respectively. On the other hand, maximum uncertainty $(\Delta \mathbf{J})^{2}$ is obtained for $\langle\mathbf{J}\rangle=\mathbf{0}$. This is the case of the state $|j, m=0\rangle$, for example (see Sec. VI).

The uncertainty relation (5.2) is nontrivial even for $\langle\mathbf{j}\rangle$ $=\mathbf{0}$. Nevertheless, this is not very informative about angular momentum statistics since this is actually just a function of the first moments $\langle\mathbf{j}\rangle$. In order to proceed further, deriving more meaningful uncertainty relations, let us split the analysis in two cases $\langle\mathbf{j}\rangle \neq \mathbf{0}$ and $\langle\mathbf{j}\rangle=\mathbf{0}$.

\section{A. Case $\langle\mathrm{j}\rangle \neq 0$}

\section{Product of variances}

For $\langle\mathbf{j}\rangle \neq \mathbf{0}$ an $\mathrm{SU}(2)$ invariant product of variances can be derived by applying the standard procedure to the longitudinal and transversal components (2.8), leading to just one nontrivial relation [12]

$$
\Delta j_{\perp, 1} \Delta j_{\perp, 2} \geqslant \frac{1}{2}\left|\left\langle j_{\|}\right\rangle\right|,
$$

while the other two are trivial $\Delta j_{\perp, k} \Delta j_{\|} \geqslant 0$, for $k=1,2$.

When $J_{\|}=j_{\|}$is a principal component we can show that the principal transversal variances $\Delta J_{\perp, k}$ provide the minimum uncertainty product 


$$
\Delta j_{\perp, 1} \Delta j_{\perp, 2} \geqslant \Delta J_{\perp, 1} \Delta J_{\perp, 2} \geqslant \frac{1}{2}\left|\left\langle j_{\|}\right\rangle\right| .
$$

This is because the determinant of $M$ is invariant under rotations of $\mathbf{j}$, and the principal components are uncorrelated. Moreover they are the extreme variances in the transversal plane according to point (vii) in Sec. III.

\section{Sum of variances}

We can begin with by particularizing Eq. (2.2) to longitudinal and transversal components leading to

$$
\left(\Delta j_{\perp, 1}\right)^{2}+\left(\Delta j_{\perp, 2}\right)^{2}=\left\langle j_{0}\left(j_{0}+1\right)\right\rangle-\left\langle j_{\|}^{2}\right\rangle .
$$

Since we have always $\left\langle j_{0}^{2}\right\rangle \geqslant\left\langle j_{\|}^{2}\right\rangle$ we get the following lower bound to the sum of transversal variances

$$
\left(\Delta j_{\perp, 1}\right)^{2}+\left(\Delta j_{\perp, 2}\right)^{2} \geqslant\left\langle j_{0}\right\rangle,
$$

where the equality is reached by the $\mathrm{SU}(2)$ coherent states exclusively.

Let us note that this relation is stronger than the similar one that can be derived from Eq. (5.4),

$$
\left(\Delta j_{\perp, 1}\right)^{2}+\left(\Delta j_{\perp, 2}\right)^{2} \geqslant\left|\left\langle j_{\|}\right\rangle\right|
$$

since $\left\langle j_{0}\right\rangle \geqslant\left|\left\langle j_{\|}\right\rangle\right|$always.

Equation (5.7) holds irrespectively of whether $j_{\|}$is a principal component or not. Furthermore, when $j_{\|}$is a principal component of $M$ or $\tilde{M}$ we have, respectively

$$
\begin{gathered}
\left(\Delta J_{\perp, 1}\right)^{2}+\left(\Delta J_{\perp, 2}\right)^{2} \geqslant\left\langle j_{0}\right\rangle, \\
\left(\Delta \tilde{J}_{\perp, 1}\right)^{2}+\left(\Delta \widetilde{J}_{\perp, 2}\right)^{2} \geqslant\left\langle j_{0}\right\rangle . \\
\text { B. Case }\langle\mathbf{j}\rangle=\mathbf{0}
\end{gathered}
$$

States with $\langle\mathbf{j}\rangle=\mathbf{0}$ arise very often in quantum metrological applications as explained in point (xi) of Sec. III $[1,20,21]$. In such a case the standard uncertainty products (5.1) are all trivial $\Delta j_{k} \Delta j_{\ell} \geqslant 0$ since they do not establish any lower bound to the product of variances. Moreover, the components $j_{\|}, j_{\perp}$ are undefined.

\section{Product of variances}

We can derive a suitable lower bound for the product $\Delta J_{1} \Delta J_{2}$ of the two larger principal variances of $M$ (or $\tilde{M}$ ) with $\Delta J_{1} \geqslant \Delta J_{2} \geqslant \Delta J_{3}$, valid for all states with $\langle\mathbf{j}\rangle=\mathbf{0}$.

To this end we begin with by considering the minimum of $\Delta J_{2}$ for fixed $\Delta J_{1}$. From the equality (3.14) we get

$$
\left(\Delta J_{2}\right)^{2}+\left(\Delta J_{3}\right)^{2}=\left\langle j_{0}\left(j_{0}+1\right)\right\rangle-\left(\Delta J_{1}\right)^{2},
$$

and for fixed $\Delta J_{1}$ the sum $\left(\Delta J_{2}\right)^{2}+\left(\Delta J_{3}\right)^{2}$ is constant. Taking into account that $\Delta J_{2} \geqslant \Delta J_{3}$ we get that the minimum $\Delta J_{2}$ occurs when

$$
\Delta J_{2}=\Delta J_{3}=\frac{1}{2}\left[\left\langle j_{0}\left(j_{0}+1\right)\right\rangle-\left(\Delta J_{1}\right)^{2}\right],
$$

and then it holds that

$$
\left(\Delta J_{2}\right)^{2}\left(\Delta J_{1}\right)^{2} \geqslant \frac{1}{2}\left(\Delta J_{1}\right)^{2}\left[\left\langle j_{0}\left(j_{0}+1\right)\right\rangle-\left(\Delta J_{1}\right)^{2}\right] .
$$

The minimum of the right-hand side takes place when $\Delta J_{1}$ reaches its extreme values in Eq. (3.15) so that

$$
\left(\Delta J_{2}\right)^{2}\left(\Delta J_{1}\right)^{2} \geqslant \min \left[\frac{1}{2}\left\langle j_{0}\right\rangle\left\langle j_{0}^{2}\right\rangle, \frac{1}{9}\left\langle j_{0}\left(j_{0}+1\right)\right\rangle^{2}\right],
$$

where "min" refers to the minimum of the alternatives.

For $\left\langle j_{0}\right\rangle \geqslant 2$ we get that this is always

$$
\left(\Delta J_{2}\right)^{2}\left(\Delta J_{1}\right)^{2} \geqslant \frac{1}{2}\left\langle j_{0}\right\rangle\left\langle j_{0}^{2}\right\rangle,
$$

and the equality is reached for states with maximum $\Delta J_{1}$ and minimum $\Delta J_{2}$,

$$
\left(\Delta J_{1}\right)^{2}=\left\langle j_{0}^{2}\right\rangle, \quad\left(\Delta J_{2}\right)^{2}=\left(\Delta J_{3}\right)^{2}=\frac{1}{2}\left\langle j_{0}\right\rangle .
$$

We will see in the next section that this is the case of the quantum superposition (so-called "Schrödinger cat") states (6.14).

\section{Sum of variances}

Nontrivial bounds to sums of variances can be derived by particularizing Eq. (2.2) to three components $j_{u}, j_{v}, j_{w}$ obtained by projection on three mutually orthogonal (complex in general) unit vectors $\mathbf{u}, \mathbf{v}, \mathbf{w}$.

$$
\left(\Delta j_{u}\right)^{2}+\left(\Delta j_{v}\right)^{2}+\left(\Delta j_{w}\right)^{2}=\left\langle j_{0}\left(j_{0}+1\right)\right\rangle .
$$

Since for any projection, say $j_{w}$, we have $\left\langle j_{0}^{2}\right\rangle \geqslant\left\langle j_{w}^{\dagger} j_{w}\right\rangle$ $=\left(\Delta j_{w}\right)^{2}$ we get

$$
\left(\Delta j_{u}\right)^{2}+\left(\Delta j_{v}\right)^{2} \geqslant\left\langle j_{0}\right\rangle
$$

where $\mathbf{u}, \mathbf{v}$ are orthogonal complex unit vectors $\mathbf{u}^{\dagger} \cdot \mathbf{v}=0$. As a byproduct we obtain that for $\langle\mathbf{j}\rangle=\mathbf{0}$ only one principal variance $\Delta \widetilde{J}$ can vanish.

Finally, we can appreciate that the above relations involve the trace of the covariance matrix being derived seemingly without resorting to commutation relations. Nevertheless, commutation relations are also at the heart of these uncertainty relations since this is the ultimate reason forbidding the simultaneous vanishing of the fluctuations of all angularmomentum components. In this regard, as shown in the original Schrödinger's paper position-momentum uncertainty relations can be fruitfully related to the corresponding covariance matrix [22].

\section{EXAMPLES}

In this section we apply the preceding formalism to some relevant and illustrative quantum states.

\section{A. States $|\mathbf{j}, \mathbf{m}\rangle$}

Let us consider the simultaneous eigenstates $|j, m\rangle$ of $j_{0}$ and $j_{3}$ with eigenvalues $j$ and $m$, respectively. This family 
includes the $\mathrm{SU}(2)$ coherent states for $m= \pm j$ and the limit of SU(2) squeezed coherent states for $m=0[1,10]$. For twomode bosonic realizations the case $m=0$ is the product of states with the same definite number of particles in each mode [20].

For $m \neq 0$ we have $\langle\mathbf{j}\rangle \neq \mathbf{0}$ and there is a longitudinal component with $j_{\|}=j_{3}$. In such a case $U \rho U^{\dagger}=\rho$ for $U$ $=\exp \left(i \pi j_{\|}\right)$and $j_{\|}$is a principal component.

The real symmetric covariance matrix is directly diagonal in any basis containing the longitudinal component $j_{3}=j_{\|}$,

$$
M=\frac{1}{2}\left(\begin{array}{ccc}
j(j+1)-m^{2} & 0 & 0 \\
0 & j(j+1)-m^{2} & 0 \\
0 & 0 & 0
\end{array}\right),
$$

with principal variances

$$
(\Delta J)^{2}=0, \frac{1}{2}\left[j(j+1)-m^{2}\right] .
$$

On the other hand, the complex Hermitian covariance matrix is

$$
\tilde{M}=\frac{1}{2}\left(\begin{array}{ccc}
j(j+1)-m^{2} & i m & 0 \\
-i m & j(j+1)-m^{2} & 0 \\
0 & 0 & 0
\end{array}\right),
$$

with principal components

$$
\begin{gathered}
\tilde{J}_{\perp, 1}=\frac{1}{\sqrt{2}} j_{+}=\frac{1}{\sqrt{2}}\left(j_{1}+i j_{2}\right), \\
\widetilde{J}_{\perp, 2}=\frac{1}{\sqrt{2}} j_{-}=\frac{1}{\sqrt{2}}\left(j_{1}-i j_{2}\right), \\
\widetilde{J}_{\|}=j_{3},
\end{gathered}
$$

so that $\widetilde{J}_{\perp, 1,2}$ are proportional to the ladder operators $j_{ \pm}$. The principal variances are

$$
\begin{gathered}
\left(\Delta \widetilde{J}_{\perp, 1}\right)^{2}=\frac{1}{2}[j(j+1)-m(m+1)], \\
\left(\Delta \widetilde{J}_{\perp, 2}\right)^{2}=\frac{1}{2}[j(j+1)-m(m-1)], \\
\left(\Delta \widetilde{J}_{\|}\right)^{2}=0,
\end{gathered}
$$

which are larger and lesser, respectively, than the variances (6.2) of the real symmetric case, in accordance with point (ix) of Sec. IV.

The $\mathrm{SU}(2)$ coherent states are minimum uncertainty states for the sum of three variances in Eq. (5.2), and for the product and sum of variances of transversal components in Eqs. (5.5), (5.7), and (5.9). The scaling of the largest principal variance as $\left(\Delta J_{1}\right)^{2} \propto j$ agrees with the fact that the $\mathrm{SU}(2)$ coherent states are not optimum for metrological applications. Optimum states scaling as $\left(\Delta J_{1}\right)^{2} \propto j^{2}$ can be found below in this section.
For $\mathrm{SU}(2)$ coherent states two of the principal variances in Eq. (6.5) vanish. This corresponds to the fact that they satisfy the double eigenvalue relation $j_{ \pm}|j, \pm j\rangle=0$ and $j_{3}|j, \pm j\rangle= \pm j|j, \pm j\rangle$, so that $|j, \pm j\rangle$ are eigenstates of two of the principal components in Eq. (6.4).

On the other hand, for the states with $m \neq \pm j$ the nonvanishing variances increase for decreasing $|m|$ and for $m=0$ the maximum scales as $\left(\Delta J_{1}\right)^{2} \propto j^{2}$, which is consistent with the usefulness of these states in quantum metrology, in agreement with points (ix) and (xi) in Sec. III [20]. Moreover, the states $m=0$ are far from the lower bounds of the uncertainty relations in Eqs. (5.2), (5.14), and (5.17).

\section{B. SU(2) squeezed coherent states}

Let us consider the SU(2) squeezed coherent states defined by the eigenvalue equations $[1,11]$

$$
\left(j_{\perp, 1}+i \xi j_{\perp, 2}\right)|\xi\rangle=0, \quad j_{0}|\xi\rangle=j|\xi\rangle,
$$

where $\xi$ is a real parameter with $\xi \geqslant 0$ without loss of generality. The first of these equations corresponds to the case of zero eigenvalue among a larger family of eigenvalue equations [1]. The states $|\xi\rangle$ are fully defined by the eigenvalue relations (6.6). An approximate solution is provided below in Eq. (6.8). For $\xi=1$ these states are the SU(2) coherent states while for $\xi \neq 1$ they are SU(2) squeezed coherent states being minimum uncertainty states of the uncertainty product (5.5) with reduced fluctuations in the component $j_{\perp, 1}$ for $\xi<1$. They satisfy the squeezing criterion suitable for interferometric and spectroscopic measurements approaching the Heisenberg limit $[1,11]$.

It is worth stressing that for any $\xi$ the vanishing of the eigenvalue in Eq. (6.6) grants that $j_{\perp, k}, k=1,2$, are actually transversal components for all parameters $\xi$. This can be readily seen by projecting Eq. (6.6) on $|\xi\rangle$.

Furthermore, we can show that Eq. (6.6) grants also that the operators $j_{\perp, k}$ in Eq. (6.6) and $j_{\|}=-i\left[j_{\perp, 1}, j_{\perp, 2}\right]$ are principal components. To this end we can project Eq. (6.6) on $j_{\|}|\xi\rangle$ leading to

$$
\left\langle\xi\left|j_{\|} j_{\perp, 1}\right| \xi\right\rangle+i \xi\left\langle\xi\left|j_{\|} j_{\perp, 2}\right| \xi\right\rangle=0 .
$$

The commutation relations and $\left\langle j_{\perp, k}\right\rangle=0$ imply that $\left\langle j_{\mid j_{\perp, k}}\right\rangle$ $=\left\langle j_{\perp, k} j_{\|}\right\rangle$so that the mean values in Eq. (6.7) are real quantities. Thus Eq. (6.7) implies that both mean values vanish and $j_{\|}$is a principal component both for $M$ and $\tilde{M}$. Moreover, by adding the projections on $j_{\perp, 1}|\xi\rangle$ and $-i \xi j_{\perp, 2}|\xi\rangle$ we get that $j_{\perp, k}$ are uncorrelated in the sense that $\langle\xi|\left(j_{\perp, 1} j_{\perp, 2}\right.$ $\left.+j_{\perp, 2} j_{\perp, 1}\right)|\xi\rangle=0$.

Therefore, $M$ is diagonal in the $j_{\perp, k}, j_{\|}$basis so they are the principal components of $M$. The vanishing of the eigenvalue in Eq. (6.6) is the only possibility dealing with principal components since otherwise the correlations between components are proportional to the eigenvalue, spoiling property (iii) in Sec. III [23].

All this suggests that Eq. (6.6) may be taken as the proper $\mathrm{SU}(2)$ invariant form of defining the $\mathrm{SU}(2)$ squeezed coherent states. The exact solution of Eq. (6.6) for arbitrary $\xi$ is difficult to handle [1]. For definiteness we can consider the 
limit $\xi \rightarrow 0$ retaining the first nonvanishing power on $\xi$. In the basis $|j, m\rangle$ of eigenvectors of $j_{0}$ and $j_{\perp, 1}$ we have

$$
|\xi\rangle \simeq N\left[|j, 0\rangle-\frac{i}{2} \xi \sqrt{j(j+1)}(|j, 1\rangle-|j,-1\rangle)\right],
$$

where $N$ is a normalization constant.

In this approximation, the principal variances of $M$ are

$$
\begin{gathered}
\left(\Delta J_{\perp, 1}\right)^{2} \simeq \frac{1}{2} j(j+1) \xi^{2}, \\
\left(\Delta J_{\perp, 2}\right)^{2} \simeq\left(\Delta J_{\|}\right)^{2} \simeq \frac{1}{2} j(j+1),
\end{gathered}
$$

with

$$
\left\langle J_{\|}\right\rangle \simeq j(j+1) \xi
$$

This is a minimum uncertainty state for the uncertainty product in Eq. (5.5) while for the sums of variances it behaves essentially as the state $|j, m=0\rangle$. The metrological usefulness of these states is confirmed by the scaling of the maximum principal variance as $\left(\Delta J_{1}\right)^{2} \propto j^{2}$, in accordance with point (ix) in Sec. III.

The complex Hermitian covariance matrix is no longer diagonal in the $j_{\perp, k}$ basis

$$
\tilde{M}=\frac{j(j+1)}{2}\left(\begin{array}{ccc}
\xi^{2} & i \xi & 0 \\
-i \xi & 1 & 0 \\
0 & 0 & 1
\end{array}\right)
$$

The principal components are

$$
\begin{gathered}
\tilde{J}_{\perp, 1}=j_{\perp, 1}+i \xi j_{\perp, 2}, \\
\tilde{J}_{\perp, 2}=j_{\perp, 2}+i \xi j_{\perp, 1}, \\
\tilde{J}_{\|}=j_{\|},
\end{gathered}
$$

with principal variances

$$
\begin{gathered}
\left(\Delta \tilde{J}_{\perp, 1}\right)^{2}=0 \\
\left(\Delta \widetilde{J}_{\perp, 2}\right)^{2} \simeq\left(\Delta \widetilde{J}_{\|}\right)^{2} \simeq \frac{1}{2} j(j+1) .
\end{gathered}
$$

The vanishing of $\Delta \widetilde{J}_{1}$ is equivalent to the eigenvalue equation (6.6).

\section{Schrödinger cat states}

In this context a suitable example of Schrödinger cat states is the coherent superposition of two opposite $\mathrm{SU}(2)$ coherent states. In the basis of simultaneous eigenvectors of $j_{0}$ and a properly chosen $j_{3}$ we have

$$
|\psi\rangle=\frac{1}{\sqrt{2}}(|j, j\rangle+|j,-j\rangle), \quad j_{0}|\psi\rangle=j|\psi\rangle,
$$

which for large $j$ are also known as maximally entangled states, or NOON states, because of their form in the number basis of two-mode bosonic realizations, being also of much interest in metrological applications [21].

For $j=1 / 2$ these are $\mathrm{SU}(2)$ coherent states while for $j$ $\geqslant 1$ we have $\langle\mathbf{j}\rangle=\mathbf{0}$ and $M$ and $\tilde{M}$ coincide,

$$
M=\tilde{M}=\left(\begin{array}{ccc}
j / 2+\delta_{j, 1} / 2 & 0 & 0 \\
0 & j / 2-\delta_{j, 1} / 2 & 0 \\
0 & 0 & j^{2}
\end{array}\right) .
$$

It can be seen that $|\psi\rangle$ is a minimum uncertainty state for the product and sum of variances in Eqs. (5.14) and (5.17) for $u, v=1,2$. On the other hand, the sum of three variances takes the maximum value possible in Eq. (5.2). Moreover, the scaling of the maximum principal variance as $\left(\Delta J_{1}\right)^{2}$ $\propto j^{2}$ confirms the metrological usefulness of these states [21].

\section{States $|j, 0\rangle+|j, 1\rangle$}

Finally, let us consider the following states expressed in the basis of eigenvectors of $j_{0}$ and $j_{3}$ as

$$
|\psi\rangle=\frac{1}{\sqrt{2}}(|j, 0\rangle+|j, 1\rangle),
$$

with applications in quantum metrology [1]. In our context these states provide an example where the longitudinal component is not principal. In this case

$$
\langle\mathbf{j}\rangle=\frac{1}{2}(\sqrt{j(j+1)}, 0,1),
$$

so that the longitudinal component is given by

$$
j_{\|}=\sin \theta j_{1}+\cos \theta j_{3}, \quad \tan \theta=\sqrt{j(j+1)} .
$$

On the other hand, $M$ is diagonal in the $\mathbf{j}$ basis,

$$
M=\frac{1}{4}\left(\begin{array}{ccc}
j(j+1)-1 & 0 & 0 \\
0 & 2 j(j+1)-1 & 0 \\
0 & 0 & 1
\end{array}\right),
$$

and

$$
\tilde{M}=\frac{1}{4}\left(\begin{array}{ccc}
j(j+1)-1 & i & 0 \\
-i & 2 j(j+1)-1 & i \sqrt{j(j+1)} \\
0 & -i \sqrt{j(j+1)} & 1
\end{array}\right),
$$

so that no principal component coincides with $j_{\|}$.

\section{CONCLUSIONS}

In this work we have elaborated the assessment of angular-momentum fluctuations via principal variances derived from the diagonalization of the covariance matrix for the problem. We have considered two forms for the covariance matrix: real symmetric and complex Hermitian. We have related the principal variances with meaningful SU(2) invariant uncertainty relations. 
In particular, we have derived nontrivial uncertainty relations for states with vanishing mean values of all angularmomentum components, for which all previously introduced variance products are trivially bounded by zero. We have found that the corresponding minimum uncertainty states are the maximally entangled states (NOON states or Schrödinger cat states). Moreover, we have demonstrated that all pure states with vanishing mean angular momentum are optimum for metrological applications since they can reach the Heisenberg limit.

\section{ACKNOWLEDGMENT}

A.L. acknowledges the support from project No. PR1-A/07-15378 of the Universidad Complutense.
[1] B. Yurke, S. L. McCall, and J. R. Klauder, Phys. Rev. A 33, 4033 (1986); M. Hillery and L. Mlodinow, ibid. 48, 1548 (1993); C. Brif and A. Mann, ibid. 54, 4505 (1996).

[2] Y. Castin and J. Dalibard, Phys. Rev. A 55, 4330 (1997); J. A. Dunningham and K. Burnett, ibid. 61, 065601 (2000); 70, 033601 (2004); V. Meyer, M. A. Rowe, D. Kielpinski, C. A. Sackett, W. M. Itano, C. Monroe, and D. J. Wineland, Phys. Rev. Lett. 86, 5870 (2001).

[3] D. J. Wineland, J. J. Bollinger, W. M. Itano, and D. J. Heinzen, Phys. Rev. A 50, 67 (1994).

[4] A. Sørensen, L.-M. Duan, J. I. Cirac, and P. Zoller, Nature (London) 409, 63 (2001); G. Tóth, Phys. Rev. A 69, 052327 (2004).

[5] N. Korolkova, G. Leuchs, R. Loudon, T. C. Ralph, and Ch. Silberhorn, Phys. Rev. A 65, 052306 (2002); N. Korolkova and R. Loudon, ibid. 71, 032343 (2005).

[6] A. Luis and L. L. Sánchez-Soto, in Progress in Optics, edited by E. Wolf (Elsevier, Amsterdam, 2000), Vol. 41, p. 421, and references therein.

[7] M. O. Scully and M. S. Zubairy, Quantum Optics (Cambridge University Press, Cambridge, England, 1997).

[8] A. Luis and L. L. Sánchez-Soto, Quantum Semiclassic. Opt. 7, 153 (1995).

[9] J. Schwinger, Quantum Theory of Angular Momentum (Academic Press, New York, 1965).

[10] F. T. Arecchi, E. Courtens, R. Gilmore, and H. Thomas, Phys. Rev. A 6, 2211 (1972).

[11] M. Kitagawa and M. Ueda, Phys. Rev. A 47, 5138 (1993).

[12] A. Luis and N. Korolkova, Phys. Rev. A 74, 043817 (2006).

[13] S. L. Braunstein and C. M. Caves, Phys. Rev. Lett. 72, 3439 (1994); S. L. Braunstein, C. M. Caves, and G. J. Milburn, Ann. Phys. 247, 135 (1996); A. Monras, Phys. Rev. A 73, 033821 (2006).
[14] J.-M. Lévy-Leblond, Ann. Phys. 101, 319 (1976); V. V. Dodonov, E. V. Kurmyshev, and V. I. Man'ko, Phys. Lett. 79A, 150 (1980).

[15] P. H. Moravek and D. W. Joseph, J. Math. Phys. 4, 1363 (1963); K. Nemoto, J. Phys. A 33, 3493 (2000); K. Nemoto and B. C. Sanders, ibid. 34, 2051 (2001).

[16] U. Leonhardt and H. Paul, Prog. Quantum Electron. 19, 89 (1995); U. Leonhardt, Measuring the Quantum State of Light (Cambridge University Press, Cambridge, England, 1997); D.-G. Welsch, W. Vogel, and T. Opatrný, in Progress in Optics, edited by E. Wolf (Elsevier Science, Amsterdam, 1999), Vol. 39.

[17] G. S. Agarwal, Phys. Rev. A 57, 671 (1998); J. P. Amiet and S. Weigert, J. Opt. B: Quantum Semiclassical Opt. 2, 118 (2000); S. Weigert, ibid. 6, 489 (2004).

[18] K. Wodkiewicz and J. H. Eberly, J. Opt. Soc. Am. B 2, 458 (1985)

[19] R. Delbourgo, J. Phys. A 10, 1837 (1977).

[20] M. J. Holland and K. Burnett, Phys. Rev. Lett. 71, 1355 (1993).

[21] N. D. Mermin, Phys. Rev. Lett. 65, 1838 (1990); J. J. Bollinger, W. M. Itano, D. J. Wineland, and D. J. Heinzen, Phys. Rev. A 54, R4649 (1996); S. F. Huelga, C. Macchiavello, T. Pellizzari, A. K. Ekert, M. B. Plenio, and J. I. Cirac, Phys. Rev. Lett. 79, 3865 (1997); A. Luis, Phys. Rev. A 64, 054102 (2001); 65, 034102 (2002); Ph. Walther, J.-W. Pan, M. Aspelmeyer, R. Ursin, S. Gasparoni, and A. Zeilinger, Nature (London) 429, 158 (2004); M. W. Mitchell, J. S. Lundeen, and A. M. Steinberg, ibid. 429, 161 (2004).

[22] E. Schrödinger, Bulg. J. Phys. 26, 193 (1999).

[23] R. N. Deb, N. Nayak, and B. Dutta-Roy, Eur. Phys. J. D 33, 149 (2005). 